\title{
Caracterización de la función cardiorrespiratoria y su relación con el estrés oxidativo en mujeres expuestas al humo de leña residentes de gran altura (3850 m s. n. m.)
}

Melgarejo-Pomar I G* 1,a; Balanza-E E 1,b; Goméz-M J 2,c; Torrez-C L d ; Riveros-L G 3,e

\section{RESUMEN}

Objetivo: Caracterizar la función cardiorrespiratoria y el estado oxidativo consecuente a la exposición al humo de leña en mujeres que viven a gran altura (3850 m s.n.m.).

Materiales y métodos: Estudio observacional serie de casos. Se exploró la función cardiorrespiratoria y se cuantificó la concentración sérica de malondialdehido (MDA) y carboxihemoglobina $(\mathrm{COHb})$ como marcadores de estrés oxidativo. Se determinaron medidas de tendencia central y de dispersión. Se aplicaron métodos estadísticos de asociación y correlación. Resultados: Se estudiaron a 60 participantes, de las cuales, 50,00 \% presentó clínica de enfermedad cardiaca; 25,00 \%, clínica de enfermedad respiratoria. El 45,00 \% de las espirometrías fueron normales; 37,67 \% mostraron disminución del volumen espiratorio forzado en un segundo (VEF 1 ), del índice de permeabilidad bronquial central (VEF $1 / \mathrm{CVF}$ ) y/o de los flujos aéreos periféricos $\left(\mathrm{FEF}_{25-75}\right) ; 8,33 \%$, disminución $\mathrm{VEF}_{1} / \mathrm{CVF} ; 3,33 \%$, disminución del $\mathrm{FEF}_{25-75} ; 1,67 \%$, disminución de la capacidad vital forzada (CVF), y 5,0\%, disminución de dos o más parámetros. El 60,00\% de los electrocardiogramas eran normales, en 36,67 \% se encontró diferentes trastornos de la conducción intraventricular, y en 3,33 \% datos de corazón dextrorotado.

El $81,67 \%$ de las ecocardiografías mostraba algún tipo de alteración: 73,33 \% evidenciaron insuficiencias valvulares; un 20,00 \% presentó, además de esta condición, hipertensión arterial; mientras que el 67,67 \%, dimensión aumentada del ventrículo derecho. La media de MDA fue de 5,62 $\mu \mathrm{M} / \mathrm{L} \pm 2,06$, correlación positiva con la edad (p: 0,029). La mediana de $\mathrm{COHb}$ fue de 1,05\% (p25:0,70-p75:1,68). No se encontró diferencia estadísticamente significativa entre la concentración de $\mathrm{COHb}$ y las alteraciones espirométricas. Se encontró correlación positiva entre los valores de MDA y el grosor del ventrículo derecho ( $p: 0,001)$.

Conclusiones: Las diferentes alteraciones de la función cardiorrespiratoria encontradas expresan que tanto la exposición crónica a los derivados del humo de leña como la hipoxia hipobárica son fuentes importantes de radicales libres y que establecen un estado permanente y sostenido de estrés oxidativo, responsable del deterioro progresivo de la función cardiorrespiratoria sin expresión clínica precoz.

Palabras clave: Estrés oxidativo; Malondialdehído; Carboxihemoglobina; Altitud (Fuente: DeCS BIREME).

\section{Characterization of the cardiorespiratory function and its relationship with oxidative stress in women exposed to wood smoke who live at high altitude $(3,850 \mathrm{~m}$ a.s.l.)}

\section{ABSTRACT}

Objective: To characterize the cardiorespiratory function and the state of oxidative stress resulting from the exposure to wood smoke in women who live at high altitude $(3,850 \mathrm{~m}$ a.s.l. $)$.

Materials and methods: An observational case-series study. The cardiorespiratory function was analyzed, and the biomarkers of oxidative stress malondialdehyde (MDA) and carboxyhemoglobin (COHb) were quantified. Measures of central tendency and dispersion were determined. The statistical methods of association and correlation were implemented.

Results: Sixty (60) patients were included in the research. Fifty percent $(50.00 \%)$ of the participants showed clinical evidence of heart disease and $25.00 \%$ had clinical symptoms of respiratory disease. Forty-five percent $(45.00 \%)$ of the spirometries revealed normal values. Thirty-six point six seven percent $(36.67 \%)$ showed a decrease in the forced expiratory volume in one second $\left(\mathrm{FEV}_{1}\right)$, forced expiratory volume in one second/forced vital capacity ratio ( $\left.\mathrm{FEV}_{1} / \mathrm{FVC}\right)$ and/or

1 Instituto Boliviano de Biología de Altura (IBBA), Facultad de Medicina, Universidad Mayor de San Andrés (UMSA). La Paz, Bolivia.

2 Instituto Nacional de Tórax. Servicio Departamental de Salud (SEDES). La Paz, Bolivia.

3 Instituto Boliviano de Biología de Altura (IBBA), Servicio Departamental de Salud (SEDES). La Paz, Bolivia.

a Especialista Neumólogo, Docente Investigador.

b Licenciada en Bioquímica, Investigador.

c Médico Especialista Cardiólogo, Ecocardiografista.

d Médico General, Investigador, Consultor.

e Técnico de laboratorio.

*Autor corresponsal. 
forced expiratory flow $25-75 \%\left(\mathrm{FEF}_{25-75}\right)$. A decrease in $\mathrm{FEV}_{1} / \mathrm{FVC}, \mathrm{FEF}_{25-75}$ and $\mathrm{FVC}$ occurred in $8.33 \%, 3.33 \%$ and $1.67 \%$ of the study participants, respectively. Moreover, a $5.00 \%$ decrease was found in more than two parameters. Sixty percent $(60.00 \%)$ of the electrocardiograms revealed normal values, $36.67 \%$ presented an intraventricular conduction disorder and $3.33 \%$ showed dextrorotation of the heart. Eighty-one point six seven percent $(81.67 \%)$ of the participants presented an echocardiographic alteration, $73.33 \%$ showed valvular insufficiency, $20.00 \%$ presented pulmonary hypertension and $6.67 \%$ had an enlarged right ventricle. The mean MDA was $6 \mu M / L \pm 2$, which was positively correlated with the age $(p: 0.029)$. The median $\mathrm{COHb}$ accounted for $1.00 \%$ (p25: 0.70 - p75: 1.68). No statistically significant association was found between the $\mathrm{COHb}$ concentration and spirometric alterations. A positive correlation was found between MDA values and the right ventricle wall thickness ( $p$ : 0.001).

Conclusions: The different alterations of the cardiorespiratory function found in the study population show that both chronic exposure to wood smoke derivatives and hypobaric hypoxia are an important source of free radicals and lead to a permanent and sustained state of oxidative stress, which is responsible for the progressive deterioration of the cardiorespiratory function with no early clinical manifestation.

Keywords: Oxidative stress; Malondialdehyde; Carboxyhemoglobin; Altitude (Source: MeSH NLM).

\section{INTRODUCCIÓN}

El último informe del Global Burden of Disease 2010 estima que la exposición al humo de leña en interiores es responsable de 3,5 millones de muertes prematuras en todo el mundo, afectando la salud de mujeres y niños, considerados población vulnerable por estar más horas expuestos a la contaminación del aire en sus hogares ${ }^{(1,2)}$.

La combustión de biomasa emite una variedad de contaminantes ambientales, los más importantes son $\mathrm{CO}$ y el material particulado (PM), este último clasificado por su tamaño en $P M<10 \mu M$ (PM10), <2,5 $\mu$ M (PM 2,5 o fino) y $<0,1 \mu \mathrm{M}$, partículas ultra finas (UFP).

Los $\mathrm{PM}_{2,5}$ y las UFP están formados, además, por metales, compuestos orgánicos e hidrocarburos policíclicos (PAH), sustancias con mayor toxicidad sistémica ${ }^{(3)}$.

Durante la combustión, también se emiten las bolas de alquitrán (hollín), partículas amorfas y carbonosas con diámetros de entre diez y cientos de nanómetros que constituyen una fracción considerable de aerosoles carbonosos que se depositan en el intersticio pulmonar ${ }^{(4)}$.

La inhalación de estos compuestos promueve la inflamación de la vía aérea, para luego establecer una inflamación sistémica. En ese momento o después se desencadena un desequilibrio entre oxidantes y antioxidantes, denominado estrés oxidativo, involucrado en la génesis de enfermedades crónicas cardiovasculares y pulmonares ${ }^{(3)}$.

La concentración de $\mathrm{PM}_{2,5}(\leq 2,5 \mu \mathrm{m})$ en el entorno interno y externo al área de combustión ha sido cuantificada en muchos estudios: se ha calculado que el promedio anual es de hasta cincuenta veces mayor al promedio aceptable por la Guía de Calidad del Aire de la OMS (gestión 2006). Esta concentración está en relación directa con la incidencia de neumonías en niños y la predisposición de contraer enfermedades como la enfermedad pulmonar obstructiva crónica (EPOC), la fibrosis pulmonar (EPID), el asma y el cáncer pulmonar en la adultez ${ }^{(5,6)}$.

Los efectos inflamatorios en el pulmón también se traducen en la alteración de la función respiratoria, principalmente en la disminución del volumen espiratorio forzado en un segundo $\left(\mathrm{VEF}_{1}\right)$ y de la capacidad vital forzada (CVF) ${ }^{(5)}$.

Respecto a la función cardiovascular, el automatismo cardiaco y la estructura miocárdica están comprometidos, siendo ambos factores de riesgo para desarrollar una enfermedad isquémica miocárdica ${ }^{(5)}$.

Otra fuente importante de radicales libres es el ambiente de hipoxia hipobárica al que se exponen cerca de 140 millones de personas que viven permanentemente a gran altura ( $\geq 2500$ m s.n.m.) ${ }^{(7)}$.

En razón de estas dos fuentes importantes de radicales libres, este estudio se realizó en un grupo de mujeres expuestas al humo de leña y residentes de gran altura (3850 m s. n. m.).

Indagamos la función cardiorrespiratoria y determinamos la concentración de malondialdehido (MDA) y el porcentaje de carboxihemoglobina como marcadores de estrés oxidativo en un grupo de mujeres del área rural del Altiplano del departamento de La Paz, Bolivia, en donde más de la mitad de las familias usa leña de eucalipto como combustible casero ${ }^{(8)}$.

\section{MATERIALES Y MÉTODOS}

\section{Diseño y población de estudio}

Estudio observacional de serie de casos con un muestreo no probabilístico, no aleatorizado, y selección por conveniencia.

El estudio original seleccionó a 133 mujeres oriundas y 
residentes de gran altura expuestas al humo de leña desde la vida intrauterina y/o por el lapso de 10 años como mínimo, cuyo rango de edad fue de 14 a 55 años. Setenta y cuatro participantes fueron subseleccionadas por considerarse libres de factores que pudiesen elevar los valores de MDA. También fueron excluidas las mujeres con antecedentes de enfermedades cardiorrespiratorias, metabólicas u otras enfermedades crónicas; posteriormente se excluyeron 14 participantes más: doce mujeres por falta de criterios de reproducibilidad y repetitividad en la espirometría, una por patología cardiaca congénita encontrada al realizar la ecocardiografía y otra por no haber aceptado realizarse la ecocardiografía.

Los datos de 60 participantes fueron incluidos en el análisis. Todos los exámenes y la toma de muestra sanguínea se realizaron en los ambientes del Hospital de Copacabana y del Centro de Salud de Siripaca en condiciones que garantizaron el bienestar de las participantes.

El estudio se realizó en la comunidad rural de Siripaca, municipio de Copacabana de la provincia Manco Kapac en el departamento de La Paz (Bolivia), ubicada a 3850 m s. n. m., a orillas del lago Titicaca. Las viviendas no cuentan con un ambiente que se emplee exclusivamente como cocina y que esté apartado de las demás habitaciones. Las paredes de las casas son de adobe, con nula o escasa ventilación. Tradicionalmente, la leña de eucalipto combustiona en fogones. El cuatro cocina alberga al núcleo familiar por muchas horas durante la cocción de los alimentos.

\section{Variables y mediciones}

Se realizó un examen clínico general, con énfasis en la esfera cardiorespiratoria, previo a la realización de las pruebas funcionales.

Se determinó la concentración plasmática de MDA y $\mathrm{COHb}$ con técnicas espectrofotométricas previamente estandarizadas.

El examen físico fue realizado por dos médicos especialistas, neumólogo y cardiólogo, respectivamente.

El peso y la talla se midieron con una balanza mecánica de pie con tallímetro modelo 2391 (DETECTO-USA), calibrada cada día.

La presión arterial fue medida con un tensiómetro marca Alpk2 300-V japonés, y tomada en el brazo izquierdo o derecho en posición decúbito.

La función pulmonar fue explorada a través de una espirometría forzada-curva flujo/volumen realizada con un espirómetro portátil Micro Loop, bajo la norma internacional de la Sociedad Española de Neumología y Cirugía Torácica (SEPAR), cumpliendo los requisitos de reproducibilidad y repetitividad. Se determinaron la capacidad vital forzada (CVF), el volumen espiratorio forzado en un segundo $\left(\mathrm{VEF}_{1}\right)$, la relación $\mathrm{VEF}_{1} / \mathrm{FVC}$ y el flujo espiratorio forzado $\mathrm{FEF}_{25-75}$.

El espirómetro fue calibrado según el manual del fabricante, evitando la presencia de maniobras incorrectas, se verificaron las condiciones ambientales, como la temperatura del ambiente $\left(37^{\circ} \mathrm{C}\right)$, presión barométrica entre 494 y $495 \mathrm{mmHg}$ y una humedad relativa de $84 \%$, utilizando una estación meteorológica portátil Perception II Station DAVIS 1991.

Se consideraron valores de referencia de Knudson, la raza no caucásica y un factor de corrección 90. Las participantes podían realizar la espirometría en posición sentada o de pie, dependiendo de la calidad de las curvas espirométricas, y debieron haber logrado por lo menos tres maniobras aceptables. Se determinó la mayor CVF y el mayor FEV ${ }_{1}$.

El electrocardiograma de superficie fue realizado según las normas de la American Heart Association, y evaluó el ritmo, la frecuencia cardiaca, el eje eléctrico, las sobrecargas auriculares y ventriculares y las alteraciones de la conducción intraventricular. Se empleó el electrocardiógrafo NIHON KOHDEN 1150 (Japón) de doce derivaciones.

La ecocardiografía se realizó con un ecocardiógrafo Doppler Color Vivid i (General Electric) con transductor multifrecuencia $3 \mathrm{~s}$ (de $3,5-7 \mathrm{mHz}$ ); de acuerdo con la normativa de la Amerian College of Echocardiografy, el procedimiento fue relizado por un médico cardiólogo subespecialista en ecocardiografía.

La muestra de sangre se tomó en la vena basílica del brazo derecho o izquierdo. Para ello, la participante debía cumplir un ayuno de 8 horas como mínimo. De los $5 \mathrm{ml}$ de sangre extraídos, $3 \mathrm{ml}$ se emplearon para cuantificar el MDA (tubo sin anticoagulante), y con los $2 \mathrm{ml}$ restantes se determinó el valor de $\mathrm{COHb}$ (tubo heparinizado).

Las técnicas para la cuantificación de MDA y $\mathrm{COHb}$ fueron previamente estandarizadas y los valores se expresaron en unidades $\mu \mathrm{Mol} / \mathrm{L}$ para MDA, y en porcentaje (\%) para COHb. Se utilizó un espectrofotómetro UV visible Evolution $60 \mathrm{~S}$ TERMOFISHER Scientific.

\section{Análisis estadístico.}

Las variables cualitativas se presentaron como frecuencias; y las cuantitativas, a través de medidas de tendencia central.

Para determinar la normalidad de las curvas de MDA y $\mathrm{COHb}$ se realizaron las pruebas de Kolmogorov Smirnov y Shapiro-Wilk, respectivamente. 
Se utilizó la prueba de t de Student-Mann Whitney para explorar diferencias, y la prueba de Spearman para establecer correlaciones.

El valor de $p<0,05$ fue aceptado como significativo. Para el análisis estadístico se empleó el programa SPSS versión 22.

\section{Consideraciones éticas}

El estudio es parte del trabajo de investigación Estrés oxidativo por uso de leña y exposición al monóxido carbonorepercusión cardiorrespiratoria en mujeres nativas y residentes de altura, cuyo protocolo de estudio fue aprobado por el Comité de Ética en Investigación de la Universidad Mayor de San Andrés, y fue financiado para su ejecución con recursos de Impuestos a los Hidrocarburos (IDH).

El consentimiento informado fue firmado por las participantes adultas mayores de 18 años; se solicitó el consentimiento de los padres o tutores para autorizar la participación de las menores de edad. Todas las participantes fueron informadas sobre los resultados e implicaciones.

\section{RESULTADOS}

Los resultados de 60 participantes fueron analizados y se identificaron dos grupos etarios: menores de 20 años y mayores de 20 (Tabla 1).

La media de MDA determinada fue de 5,62 $\mu \mathrm{M} / \mathrm{L} \pm 2,06$ con una correlación débil positiva entre los grupos etarios, con Rho Spearman + 0,28 ( $p: 0,029)$. La mediana de carboxihemoglobina fue de 1,05 \% (p25-p75: 0,70-1,68) (Tabla 1).

La media de MDA determinada fue de 5,62 $\mu \mathrm{M} / \mathrm{L} \pm 2,06$ con una correlación débil positiva entre los grupos etarios, con Rho Spearman + 0,28 ( $p: 0,029)$. La mediana de carboxihemoglobina fue de 1,05\% (p25-p75: 0,70-1,68) (Tabla 1).

Tabla 1. Grupos etarios: valores de MDA y $\mathrm{COHb} \%$

\begin{tabular}{lcccc}
\multicolumn{1}{|c}{ Grupos etario } & $\mathrm{n}$ & $\begin{array}{c}\mathrm{MDA} \mu \mathrm{M} / \mathrm{L} \\
\mathrm{X}(\overline{\mathrm{DE}})\end{array}$ & $p$ & $\begin{array}{c}\mathrm{COHb} \% \\
\mathrm{M}_{\mathrm{e}}\left(p_{25}-p_{75}\right)\end{array}$ \\
\hline $\begin{array}{l}\text { Menores de } 20 \\
\text { años }\end{array}$ & 23 & $5,11(1,79)$ & & $0,8(0,6-1,4)$ \\
$\begin{array}{l}\text { Mayores de } 20 \\
\text { años }\end{array}$ & 37 & $5,94(2,18)$ & 0,131 & $1,2(0,8-1,9)$ \\
\end{tabular}

El 56,66 \% de las participantes cursaron el nivel secundario (Tabla 2).

Tabla 2. Nivel escolar

\begin{tabular}{|llc|}
\hline Nivel escolar & n & $\%$ \\
\hline Primaria & 24 & 40,0 \\
\hline Secundaria & 34 & 56,6 \\
\hline Técnico Medio & 1 & 1,67 \\
\hline Ninguno & 1 & 1,67 \\
\hline
\end{tabular}

El examen físico reveló manifestaciones clínicas en el $25,00 \%$ de las participantes con signos de obstrucción bronquial $\mathrm{y} / \mathrm{o}$ de sufrimiento intersticial como estertores secos y signo de velcro.

En el examen cardiológico, el 50,00 \% presentó reforzamiento del segundo ruido en su componente pulmonar y 11,67\% de ellas tenía soplo sistólico tricúspideo.

El 55,00 \% de las espirometrías mostraban alteraciones, la más frecuente fue la limitación ventilatoria obstructiva central y periférica (LVOCP) (Tabla 3). 
Tabla 3. Alteraciones espirométricas - concentración de MDA y \% de COHb

\begin{tabular}{llllll} 
Espirometría & $n$ & $\%$ & $\begin{array}{c}\text { MDA } \mu M / L \\
\bar{X}(\mathrm{DE})\end{array}$ & $\begin{array}{c}\text { COHb \% } \\
M_{\mathrm{e}}\left(p_{25}-p_{75}\right)\end{array}$ & $p$ \\
Normal & 27 & 45,0 & $5,84(1,89)$ & $1(0,6-1,4)$ & \\
\hline LVOC $^{*}$ & 5 & 8,33 & $4,56(0,90)$ & $1,4(0,8-1,7)$ & \\
LVOP* $^{* *}$ & 2 & 3,33 & $6,5(2,40)$ & $0,9(0,9-0,9)$ & \\
LVOCP** & 22 & 36,67 & $5,22(2,25)$ & $1,1(0,7-1,9)$ & 0,634 \\
\hline LVR $^{* * * *}$ & 1 & 1,67 & $10,7(-)$ & $1,2(1,2-1,2)$ & \\
\hline LV Mixta**** $^{*}$ & 3 & 5,0 & $6,2(1,48)$ & $1,1(0,4-2,2)$ & \\
TOTAL & 60 & 100 & $5,62(2,06)$ & $1,05(0,70-1,68)$ &
\end{tabular}

*LVOC: Limitación ventilatoria obstructiva central (disminución del flujo espiratorio forzado en un segundo (FEV 1 ) y disminución del cociente flujo espiratorio forzado en un segundo sobre volumen espiratorio forzado ( $\mathrm{FEV}_{1} / \mathrm{FVC}$ ).

**LVOP: Limitación ventilatoria obstructiva periférica (disminución de los flujos espiratorios periféricos (FEF 25\%, FEF 50\%, FEF 75\%)

***LVOCP: Limitación ventilatoria obstructiva central y periférica

****LVR: Limitación ventilatoria restrictiva (disminución de la capacidad vital forzada FVC)

*****LVM: Limitación ventilatoria mixta (disminución del FVC y FEV 1 , FEV $1 / \mathrm{FVC}_{\text {y }} \mathrm{FEF}_{25-75} \%$.

En la participante con LVR se encontró un valor de 10,7 $\mu \mathrm{M} / \mathrm{L}$ de MDA, además, esta paciente también presentó BCRD e IT-IM-HAP-FSVDD-DVDA-DADA-TAPD. Se halló un valor de $1,4 \%$ de $\mathrm{COHb}$ en las participantes con LVOC en la espirometría (Tabla 3).

Asimismo, no existe diferencia estadísticamente significativa entre el porcentaje de $\mathrm{COHb}$ y la presencia de alteraciones en la espirometría Mann-Whitney; (p: 0,634) (Tabla 3).

El $60,00 \%$ de las participantes no mostró alteración en el electrocardiograma (ECG); 13,33\%presentóritmo de bradicardia sinusal (BS); otro 13,33\%, hemibloqueo anterosuperior izquierdo (HBASI); y el 13,34\%, un tipo distinto de alteraciones. Además, el valor más elevado de $\mathrm{COHb}$ de $3,9 \%$ se encontró en una participante con BIRD (Tabla 4).

Tabla 4. Alteraciones electrocardiográficas-MDA y $\mathrm{COHb}$

\begin{tabular}{lllll} 
& $n$ & $\%$ & $\begin{array}{c}\text { MDA } \mu M / L \\
\times(D E)\end{array}$ & $\begin{array}{c}\text { COHb \% } \\
M_{e}\left(p_{25}-p_{75}\right)\end{array}$ \\
\hline ECK Normal & 36 & 60,0 & $5,55(1,80)$ & $1(0,6-1,7)$ \\
BS & 8 & 13,33 & $5,99(1,72)$ & $0,9(0,5-1,3)$ \\
HBASI & 8 & 13,33 & $5,62(2,65)$ & $1,2(0,9-1,8)$ \\
\hline BCRD & 1 & 1,67 & $10,7(-)$ & $1,2(1,2-1,2)$ \\
\hline BIRD & 1 & 1,67 & $4,5(-)$ & $3,9(3,9-3,9)$ \\
\hline BS y HBASI & 2 & 3,33 & $5,0(2,83)$ & $1,2(1-1,4)$ \\
\hline HBVD & 1 & 1,67 & $2,4(-)$ & $0,8(0,8-0,8)$ \\
\hline BS y HBVD & 1 & 1,67 & $2,4(-)$ & $1,4(1,4-1,4)$ \\
\hline CD & 2 & 3,33 & $7,45(1,63)$ & $2,6(0,2-4,9)$ \\
\hline TOTAL & 60 & 100 & $5,62(2,06)$ & $1(0,7-1,6)$ \\
\hline
\end{tabular}

BS: Bradicardia sinusal

HBASI: Hemibloqueo anterosuperior izquierdo BCRD: Bloqueo completo de rama derecha del haz de His
BIRD: Bloqueo incompleto de rama derecha del haz de His HBVD: Hipertrofia basal de ventrículo derecho CD: Corazón dextrorotado 
El 82,00 \% de las participantes presentó alteración ecocardiográfica. De ellas, el 92,00 \% tenía insuficiencias valvulares de carácter funcional (Tabla 5).

Tabla 5. Hallazgos ecocardiográficos - valores de MDA y de CHOb\%

\begin{tabular}{|c|c|c|c|c|}
\hline & $\mathrm{n}$ & $\%$ & $\begin{array}{c}M D A \mu M / L \\
\bar{X}(D E)\end{array}$ & $\begin{array}{c}\text { COHb \% } \\
M_{e}\left(p_{25}-p_{75}\right)\end{array}$ \\
\hline Eco- Normal & 11 & 18 & $5(1)$ & $0,9(0,6-2,2)$ \\
\hline IT & 16 & 26,67 & $6(2)$ & $1,1(0,9-1,5)$ \\
\hline IM & 1 & 1,67 & $9(-)$ & $0,1(0,1-0,1)$ \\
\hline IT y IP & 7 & 11,67 & $6(1)$ & $0,7(0,6-1,7)$ \\
\hline IP & 5 & 8,33 & $4(2)$ & $0,8(0,8-1)$ \\
\hline IT y HAP & 6 & 10,0 & $6(2)$ & $1,8(0,3-2,2)$ \\
\hline DVDA & 4 & 6,67 & $4(3)$ & $1,2(0,8-2,3)$ \\
\hline IT y IM & 1 & 1,67 & $8(-)$ & $1,4(1,4-1,4)$ \\
\hline IT y DVDA & 1 & 1,67 & $8(-)$ & $2(2-2)$ \\
\hline IT, HAP y DVDA & 2 & 3,33 & $5(4)$ & $0,8 \quad(0,7-0,9)$ \\
\hline DDVI-G1 & 1 & 1,67 & $8(-)$ & $0,9(0,9-0,9)$ \\
\hline IT,IM y HAP & 1 & 1,67 & $8(-)$ & $0,2(0,2-0,2)$ \\
\hline IT-IM-HAP-FSVDD-DVDA-DADA-TAPD & 1 & 1,67 & & $1,2(1,2-1,2)$ \\
\hline IT, IP y HAP & 1 & 1,67 & $4(-)$ & $0,8(0,8-0,8)$ \\
\hline IT y IAo & 1 & 1,67 & $5(-)$ & $2,2(2,2-2,2)$ \\
\hline IT-IP-HAP-lao. & 1 & 1,67 & $4(-)$ & $1,4(1,4-1,4)$ \\
\hline TOTAL & 60 & 100 & $5,6(2)$ & $1(0,7-1,6)$ \\
\hline
\end{tabular}

IT: Insuficiencia tricúspidea

IM: Insuficiencia mitral

IP: Insuficiencia pulmonar

HAP: Hipertensión arterial pulmonar

DVDA: Dimensión de ventrículo derecho aumentado

IM: Insuficiencia mitral

Se encontró una correlación positiva moderada entre los valores de MDA y el aumento del grosor de la pared libre del ventrículo derecho. Spearman: + 0,43; $(p: 0,001)$.

Una participante con LVOP con HBASI registró DDVI-G1 en la ecocardiografía. Asimismo, el 13,64\% de las participantes con LVOCP tenían BS; y el 13,64 \%, HBASI. Tres participantes con LVM presentaron DVDA, BS, IT y HAP.
DDVI-G1: Disfunción diastólica de ventrículo izquierdo -G 1 FSVDD: Función sistólica del ventrículo derecho disminuido DADA: Dimensión de aurícula derecha aumentado TAPD: Tronco de la arteria pulmonar dilatado IAo: Insuficiencia de válvula aórtica

Doce participantes tuvieron hipertensión pulmonar (HTP): once en grado leve $(35-50 \mathrm{mmHg}$ ) y una en grado moderado (51-90 $\mathrm{mmHg})$. En diez pacientes de este grupo la espirometría mostró LVOCP. Además, no existe asociación entre los valores de MDA y la evidencia ecocardiográfica de HTP (t de Student; p: 0,194) (Tabla 6).

Tabla 6. Relación entre el grado de hipertensión pulmonar y las alteraciones espirométricas

\begin{tabular}{|cccccccr} 
& \multicolumn{4}{c}{ Alteraciones espirométricas } & \multirow{2}{*}{ Total } \\
\cline { 2 - 6 } Grados - HTP & LVOC & LVOCP & LVR & LV Mixta & Normal & \\
\cline { 1 - 6 } Leve & 2 & 6 & 0 & 1 & 2 & 11 \\
\hline Moderada & 0 & 0 & 1 & 0 & 0 & 1 \\
\hline Total & 2 & 6 & 1 & 1 & 2 & 12 \\
\hline
\end{tabular}


Caracterización de la función cardiorrespiratoria y su relación con el estrés oxidativo en mujeres expuestas al humo de leña residentes de gran altura (3850 m s. n. m.)

\section{DISCUSIÓN}

Está comprobado que la exposición crónica a los productos derivados de la combustión de leña o biomasa y a la hipoxia hipobárica constituyen factores de riesgo para enfermedades respiratorias y cardiovasculares a partir del estímulo inflamatorio generado por acción del estrés oxidativo ${ }^{(1)}$.

En Bolivia, el $28 \%$ de la población vive en el Altiplano, a una altura media de $3700 \mathrm{~m} \mathrm{s.} \mathrm{n.} \mathrm{m.,} \mathrm{y} \mathrm{se} \mathrm{expone} \mathrm{crónicamente}$ a la hipoxia hipobárica, la cual es una fuente importante de radicales libres. Considerando este importante factor de riesgo para la salud cardiorrespiratoria, el estudio fue realizado en un grupo poblacional que vive a $3850 \mathrm{~m} \mathrm{~s} . \mathrm{n}$. m., considerada como "gran altura".

Muchas especies de animales han logrado una adaptación genética al ambiente de altura; sin embargo, el hombre solo ha conseguido adaptar mecanismos fisiológicos y bioquímicos para tolerar el ambiente de hipoxia, excepto algunos grupos étnicos como los sherpas y los tibetanos. Los efectos adversos son tolerados hasta una determinada edad en la que se encuentran indemnes los mecanismos de defensa antioxidantes; luego de ese tiempo, los nativos y residentes de altura pueden presentar enfermedades consecuentes a la desadaptación que involucran las funciones del cerebro, corazón y pulmón ${ }^{(9)}$.

En Bolivia, tal como ocurre en otros países y culturas, la mujer que vive en el área rural tiene un papel doméstico muy marcado. Este factor, junto a la pobreza, las tradiciones y el bajo nivel de educación, es determinante en la persistencia del uso de la leña, la falta de comprensión del efecto nocivo del humo y sus consecuencias en la salud de las mujeres y sus familias ${ }^{(10)}$.

En Siripaca, comunidad donde se realizó el estudio, las familias usan leña de eucalipto, material fresco y húmedo que al combustionar emite gran cantidad de UFP, las que evaden el sistema mucociliar y se depositan en la vía aérea periférica, ocasionando daños permanentes sumativos en el tiempo y sin expresión clínica precoz ${ }^{(11)}$.

En cuestión de horas o días la inhalación de los productos de combustión induce a la inflamación pulmonar y, por la acción de los radicales libres, establece un estado de estrés oxidativo que conduce rápidamente a una inflamación sistémica ${ }^{(12)}$.

Existen estudios en poblaciones de altura que demuestran que la concentración de MDA es mayor en ambientes de hipoxia hipobárica, siendo la fuente de radicales libres precisamente la condición de hipoxia ${ }^{(13)}$.

Así mismo, un estudio anterior en el mismo grupo poblacional estableció que la concentración de MDA es mayor en mujeres que viven a gran altura y que se exponen al humo de leña ${ }^{(14)}$.

Los patrones espirométricos encontrados y los valores de MDA de este estudio expresan indirectamente la acción del estrés oxidativo y la acción citotóxica de los derivados de la combustión de leña en el aumento la producción de citocinas proinflamatorias como la IL-1B, IL-8 y el TNF-a, lo que al final daña al conjunto de la estructura pulmonar y el intersticio pulmonar, acción que depende del tiempo de exposición y que se evidenció principalmente en la presencia de patrones espirométricos obstructivos ${ }^{(15,16)}$.

Una proporción importante de participantes mostró disminución de los flujos espiratorios periféricos (FEF 25-75\%), patrón típico de la fibroantracosis bronquial, entidad estrechamente asociada a exposición de los aerosoles de hollín, material que se deposita en las paredes y techo de las viviendas después de cada combustión. La fibroantracosis se caracteriza por ausencia de síntomas y cambios en la función pulmonar que dependen del tiempo y la cantidad de exposición (16-18).

Es importante hacer notar que las mujeres tienen mayor susceptibilidad a desarrollar enfermedades pulmonares crónicas como la EPOC por factores anatómicos, genéticos y hormonales. Existe evidencia de que niñas expuestas a contaminantes ambientales expresan precozmente deterioro en la función pulmonar por la acción estrogénica en el aumento de la expresión de la enzima P450, encargada de activar compuestos oxidados; si bien no se realizó diagnóstico específico de EPOC, la evidencia de obstrucción bronquial en la espirometría, sobre todo la limitación al flujo de aire en bronquios de pequeño calibre, puede ser evidencia de un cuadro de EPOC fenotipo leña (EPOC-L) ${ }^{(10,19)}$.

Solo una participante presentó en la espirometría un patrón restrictivo y la concentración más elevada de MDA, este incremento revela inflamación del intersticio pulmonar como consecuencia de la acción tóxica del humo de leña ${ }^{(20)}$.

Los resultados de la concentración de $\mathrm{COHb}$ encontrados son considerados normales incluso entre personas que fuman; sin embargo, no debemos dejar de mencionar que la toma de muestra fue dos horas después de la exposición aguda al humo. Considerando que la vida media de la $\mathrm{COHb}$ en aire ambiente es de 320 minutos, ese tiempo habría sido suficiente para que un importante porcentaje de $\mathrm{CO}$ se haya separado de la hemoglobina ${ }^{(21)}$.

En este estudio, la determinación exacta de la frecuencia cardiaca, a través de la electrocardiografía, ha sido utilizada como biomarcador de disfunción autonómica. Se ha evidenciado el efecto tóxico del $\mathrm{CO}$ en la actividad eléctrica del corazón y se ha encontrado un número importante de mujeres con bradicardia sinusal y TCIV, 
cuyo origen, aunque no lo determinamos, es un desorden subclínico: la arterioesclerosis, producto de la acción infamatoria crónica en el endotelio vascular ${ }^{(3,22,23)}$.

La acción del estrés oxidativo en la remodelación y rigidez en la estructura cardiaca se advierte en los resultados del estudio ecocardiográfico al observar un aumento en el diámetro del ventrículo derecho y disfunción diastólica del ventrículo izquierdo, el primero considerado factor de riesgo para enfermedad isquémica de izquierdo ${ }^{(23-25)}$.

Respecto a los hallazgos ecocardiográficas compatibles con HTP, debemos indicar que el nativo de la altura, como mecanismo adaptativo a la hipoxia hipobárica, presenta un estado permanente de hipoxia alveolar, con el aumento leve de la presión media en la arteria pulmonar, condición conocida como como hipertensión pulmonar a gran altura (HAPH), cuyo desarrollo está asociada a la acción del estrés oxidativo en la remodelación vascular pulmonar, la neomuscularización de vasos distales y el engrosamiento de la adventicia ${ }^{(26)}$.

Un número importante de participantes presentó HTP, aunque en su mayoría de grado leve. El diagnóstico muestra que esta enfermedad está relacionada con los hallazgos espirométricos que evidencian un proceso de angiogénesis anormal en los pulmones, ya sea EPOC o fibrosis pulmonar. Se sabe que la lesión vascular pulmonar única o repetitiva, secundaria a los efectos vasoconstrictores constantes y excesivos de los derivados del humo de leña, daña el endotelio, con la consecuente remodelación de la arteria pulmonar y el aumento de la poscarga en el ventrículo derecho (VD) ${ }^{(27-29)}$.

Los resultados de este estudio caracterizan la función cardiorespiratoria de un grupo de mujeres expuestas a dos importantes condiciones ambientales: la exposición al humo de leña y la hipoxia hipobárica, y ambas establecen un estado permanente de estrés oxidativo responsable de las diferentes alteraciones en la función cardiorrespiratoria encontrados. Los hallazgos evidencian enfermedades sin manifestación clínica precoz, que tienen impacto en esta población considerada vulnerable por las condiciones sociales, culturales y económicas que la obligan a exponerse diariamente a la contaminación del aire por humo de leña.

Se recomienda la creación de políticas de alfabetización sanitaria dirigidas a grupos vulnerables como el intervenido, de tal manera que se genere una especie de empoderamiento relacionado al conocimiento de las nuevas enfermedades pulmonares y cardiovasculares que son consecuencia de la exposición crónica al humo de leña y a la altura. Es urgente la gestión de políticas sanitarias sostenibles que mejoren la calidad del aire interior en las viviendas, y también proporcionar acceso gratuito a un combustible menos nocivo como el gas.
Contribuciones de los autores: IGMP: Generación y diseño del estudio, el análisis y la interpretación de los datos, redacción de los borradores y documento final, aprobación de la versión final del estudio.

EBE: Generación y diseño del estudio, el análisis, la interpretación de los datos, redacción de los borradores y análisis del contenido de la versión final del estudio.

JSG: Aportes en el contenido del artículo.

LTC: Descripción y contenido del artículo, análisis de datos y revisión de la versión final.

LRG: Logística y realización de electrocardiogramas.

Fuentes de financiamiento: Estudio financiado por la Universidad Mayor de San Andrés, los recursos provienen del Impuesto Directos a los Hidrocarburo (IDH) obtenidos a través de concurso.

Conflicto de interés: Los autores declaran no tener ningún conflicto de interés.

\section{REFERENCIAS BIBLIOGRÁFICAS}

1. Pope D, Diaz E, Smith-Sivertsen T, Lie RT, Bakke P, Balmes JR, et al. Exposure to household air pollution from wood combustion and association with respiratory symptoms and lung function in nonsmoking women: Results from the RESPIRE trial, Guatemala. Environ Health Perspect. 2015; 123(4): 285-92.

2. Smith-Sivertsen T, Díaz E, Pope D, Lie RT, Díaz A, McCracken J, et al. Effect of reducing indoor air pollution on women's respiratory symptoms and lung function: the RESPIRE Randomized Trial, Guatemala. Am J Epidemiol. 2009; 170(2): 211-20.

3. Zhang J, Zhu T, Kipen H, Wang G, Huang W, Rich D, et al. Cardiorespiratory biomarker responses in healthy young adults to drastic air quality changes surrounding the 2008 Beijing Olympics. Res Rep Health Eff Inst. 2013; (174): 5-174.

4. Pardo M, Li C, He Q, Levin-Zaidman S, Tsoory M, Yu Q, et al. Mechanisms of lung toxicity induced by biomass burning aerosols. Part Fibre Toxicol. 2020; 17(1): 4.

5. Farmer SA, Nelin TD, Falvo MJ, Wold LE. Ambient and household air pollution: Complex triggers of disease. VAm J Physiol Heart Circ Physiol. 2014; 307(4): H467-76.

6. Kodgule R, Salvi S. Exposure to biomass smoke as a cause for airway disease in women and children. Curr Opin Allergy Clin Immunol. 2012; 12(1): 82-90.

7. Debevec T, Millet GP, Pialoux V. Hypoxia-induced oxidative stress modulation with physical activity. Front Physiol. 2017; 8: 84.

8. Instituto Nacional de Estadística. Necesidades Básicas Insatisfechas [Internet]. INE: Bolivia; 2019. Disponible en: https://www.ine.gob. bo/index.php/podreza-desarrollo/introduccion.

9. Horscroft JA, Kotwica AO, Laner V, West JA, Hennis PJ, Levett DZH, et al. Metabolic basis to sherpa altitude adaptation. Proc Natl Acad Sci U S A . 2017; 114(24): 6382-7.

10. Torres-Duque CA, García-Rodriguez MC, González-García M. Enfermedad pulmonar obstructiva crónica por humo de leña: ¿un fenotipo diferente o una entidad distinta?. Arch Bronconeumol. 2016; 52(8): 425-31.

11. Naeher LP, Brauer M, Lipsett M, Zelikoff JT, Simpson CD, Koenig JQ, et al. Woodsmoke health effects: A review. Inhal Toxicol. 2007; 19(1): 67-106.

12. Yagi K. Lipid peroxides and related radicals in clinical medicine. Adv Exp Med Biol. 1994; 366: 1-15. 
13. Melgarejo-Pomar IG, Balanza-Erquicia E, Torrez-Colmena L, Quisberth-Barrera S, Suzaño-Vargas PL. Concentración de malondialdehido, en sujetos residentes de gran altura: estudio exploratorio. Rev Peru Med Exp Salud Publica. 2017; 34(4): 677-81.

14. Melgarejo-Pomar IG, Balanza-Erquicia E, Gómez-Mendivil JS, TorrezColmena L. Estrés oxidativo por humo de leña en mujeres nativas de gran altura - 3850 m s. n. m. Horiz Med. 2020; 20(1): 61-8.

15. Naeher LP, Brauer M, Lipsett M, Zelikoff JT, Simpson CD, Koenig JQ, et al. Woodsmoke Health Effects: A Review. Inhal Toxicol. 2007; 19(1): 67-106.

16. Sada-Ovalle I, Ocaña-Guzmán R, Torre-Bouscoulet L. Humo de biomasa, inmunidad innata y Mycobacterium tuberculosis. Neumol Cir Torax. 2015; 74(2): 118-26.

17. Gupta A, Shah A. Bronchial anthracofibrosis: An emerging pulmonary disease due to biomass fuel exposure. Int J Tuberc Lung Dis. 2011; 15(5): 602-12.

18. Frampton MW. Systemic and cardiovascular effects of airway injury and inflammation: Ultrafine particle exposure in humans. Environ Health Perspect. 2001; 109(Suppl 4): 529-32.

19. Alonso T, Sobradillo P, de Torres JP. Enfermedad pulmonar obstructiva crónica en mujeres: ¿somos diferentes?. Arch Bronconeumol. 2017; 53(4): 222-7.

20. Gupta D, Agarwal R, Aggarwal AN, Maturu VN, Dhooria S, Prasad KT, et al. Guidelines for diagnosis and management of chronic obstructive pulmonary disease: Joint ICS/NCCP (I) recommendations. Lung India. 2013; 30(3): 228-67.

21. Riojas-Rodríguez H, Romieu I, Holguin F, González-Hermosillo A. Use of heartrate variability as a marker of cardiovascular effects associated with air pollution. Salud Publica Mex. 2006; 48(4): 348-57.

22. Salih $\mathrm{S}$ Bin, Alenezi $\mathrm{H}$, Alghamdi A. A case of first degree AV block in carbon monoxide poisoning patient. J Saudi Hear Assoc. 2013; 25(4): 255-9.

23. Burroughs Peña MS, Velazquez EJ, Rivera JD, Alenezi $F$, Wong $C$, Grigsby $M$, et al. Biomass fuel smoke exposure was associated with adverse cardiac remodeling and left ventricular dysfunction in Peru. Indoor Air. 2017; 27(4): 737-45.

24. Hamanaka RB, Mutlu GM. Particulate Matter Air Pollution: Effects on the Cardiovascular System. Front Endocrinol (Lausanne). 2018; 9: 680.

25. Gorr MW, Falvo MJ, Wold LE. Air pollution and other environmental modulators of cardiac function. Compr Physiol. 2017; 7(4): 1479-95.

26. Robinson JC, Abbott C, Meadows CA, Roach RC, Honigman B, Bull TM. Long-Term Health Outcomes in High-Altitude Pulmonary Hypertension. High Alt Med Biol. 2017; 18(1): 61-6.

27. Kim D, Chen Z, Zhou L-F, Huang S-X. Air pollutants and early origins of respiratory diseases. Chronic Dis Transl Med. 2018; 4(2): 75-94.

28. Frump AL, Bonnet $S$, de Jesus Perez VA, Lahm T. Emerging role of angiogenesis in adaptive and maladaptive right ventricular remodeling in pulmonary hypertension. Am J Physiol Lung Cell Mol Physiol. 2018; 314(4): L443-60.

29. Sydykov A, Mamazhakypov A, Petrovic A, Kosanovic D, Sarybaev AS, Weissmann $\mathrm{N}$, et al. Inflammatory mediators drive adverse right ventricular remodeling and dysfunction and serve as potential biomarkers. Front Physiol. 2018; 9: 609.

\section{Correspondencia:}

Ingrid Gaby Melgarejo Pomar.

Dirección: Instituto Boliviano de Biología de Altura IBBA. La Paz, Bolivia.

Teléfono: 591 - 22242059

Correo electrónico:ingridmeldany@gmail.com

igmelgarejo@umsa.bo

Revisado: 12 de febrero de 2021

Evaluado: 02 de marzo de 2021

Aprobado: 22 de marzo de 2021

( ) La revista. Publicado por Universidad de San Martín de Porres, Perú. (c) ${ }_{\text {BY }}$ Licencia de Creative Commons Artículo en acceso abierto bajo términos de Licencia Creative Commons Atribución 4.0 Internacional. (http://creativecommons.org/licenses/by/4.0/)

\section{ORCID iDs}

Ingrid Gaby Melgarejo-Pomar Th https://orcid.org/0000-0002-2485-9894

Elfride Balanza-Erquicia \ https://orcid.org/0000-0002-2128-3973

Jesús Santiago Gómez-Mendivil ㄴ https://orcid.org/0000-0002-3219-0190

Lizeth Torrez-Colmena C https://orcid.org/0000-0002-8784-8834

Loyola Riveros Gonzales $\quad$ https://orcid.org/0000-0002-2899-1921 